\title{
La criminalización de la protesta social: Un estado de la cuestión
}

Recibido: 19 de julio 2019

Revisado: 30 de agosto 2019

Aprobado: 19 de octubre 2019

\section{Alejandro Alvarado}

Alcázar

Costarricense. MSc. en

Sociología. Docente e investigador, Escuela de Sociología e Instituto de Investigaciones Sociales, Universidad de Costa Rica.

Entre sus intereses de investigación se encuentran los conflictos, movimientos y protestas sociales.

Correo electrónico: omar.alvaradoalcazar@ucr.a

c.cr

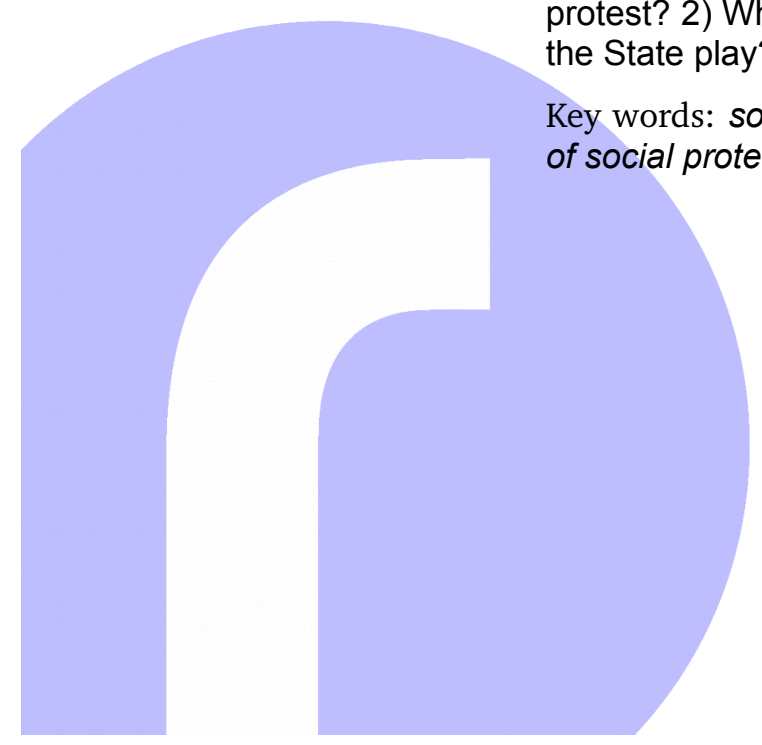

https://revistas.uned.ac.cr/index.php/rupturas/ c) (1) (2)
Resumen: Recientemente, la criminalización de la protesta se ha colocado como un tópico de discusión académica y no académica en América Latina. Pese a esto, el debate se ha desarrollado, en muchos casos, en medio de una indefinición conceptual sobre lo que es (y lo que no es) la criminalización de la protesta. El presente artículo se propone contribuir a esta discusión mediante la construcción de un estado de la cuestión que recupere los principales trabajos desarrollados en torno a este tema, poniendo particular énfasis en lo producido para el caso latinoamericano. La información ha sido obtenida mediante la consulta sistemática de bases de datos bibliográficas y se ha ordenado tratando de contestar tres preguntas fundamentales: 1) ¿qué es la criminalización de la protesta?; 2) ¿cuáles son sus manifestaciones más comunes?; y, 3) ¿qué papel juega el Estado?

Palabras clave: movimientos sociales; acción colectiva; protesta social; criminalización de la protesta; represión

\section{The Criminalization of Social Protest: A State of the Question}

Abstract: Recently, the criminalization of protest has been placed as a topic of academic and non-academic discussion in Latin America. Despite this, the debate has developed, in many cases, in the midst of a conceptual uncertainty about what is (and what is not) the criminalization of protest. The present article intends to contribute to this discussion through the construction of a state of the question that recovers the main works developed around this topic, placing particular emphasis on what has been produced for the Latin American case. The information has been obtained through the systematic consultation of bibliographic databases and it has been ordered trying to answer three fundamental questions: 1) what is the criminalization of the protest? 2) What are its most common manifestations? and, 3) what role does the State play?

Key words: social movements; collective action; social protest; criminalization of social protest; repression 
El presente trabajo es parte de los resultados de la actividad de investigación

"Criminalización contra los movimientos socioterritoriales en Costa Rica: un estado de la cuestión"; inscrita en el Instituto de Investigaciones Sociales de la Universidad de Costa Rica (IIS-UCR).

\section{Introducción. Las luchas socioterritoriales y la criminalización de} las personas defensoras de la tierra y el medio ambiente

Diferentes estudios coinciden en destacar la peligrosidad de protestar y defender los derechos humanos en América Latina, particularmente entre aquellas organizaciones y/o movimientos sociales dedicados a la defensa de la tierra y el medio ambiente (Global Witness 2015, 2016, 2017, 2018 y 2019; CIDH 2015).

Recientemente, el concepto de criminalización de la protesta ha sido usado por organismos nacionales e internacionales dedicados a la protección de los derechos humanos y por las propias organizaciones y movimientos sociales para nombrar a un conjunto de estrategias recurridas por actores estatales y no-estatales como una forma de intimidar, inhibir y deslegitimar este tipo de luchas.

Se trata de un fenómeno multidimensional que articula diferentes estrategias políticas, jurídicas y mediáticas para combatir las luchas sociales. Entre tales estrategias, el asesinato de personas defensoras de la tierra y el medio ambiente figura como la forma más descarnada (ver tabla 1); tiene una alta incidencia en el caso de América Latina, la cual se ha configurado como la región más peligrosa del mundo para la defensa de los derechos humanos.

Según datos de Global Witness, entre 2014 y 2018 fueron asesinadas 536 personas defensoras de la tierra y el medio ambiente en América Latina (ver tabla 1), cifra que representó $61,5 \%$ de todas las muertes documentadas por la organización en dicho período. Países como Brasil, Colombia, Honduras, Guatemala y México se sitúan como lugares donde defender la tierra y el medio resulta sumamente peligroso.

Todo esto ocurre en el marco de un proceso de escala regional de luchas y conflictos contra proyectos extractivos y neoextractivos, basados en un patrón de acumulación por desposesión (Harvey 2004), que se han ceñido particularmente sobre comunidades-territorios negros, indígenas y campesinos, que se han articulado en luchas de tipo socioterritorial, para tratar de frenar la desposesión de sus territorios. Este proceso, registrado desde finales de la década de 1990, se ha intensificado durante las primeras dos décadas de este siglo, configurando un ciclo de luchas socioterritoriales con una notable cantidad de manifestaciones a escala local y regional (Navarro y Pineda 2009; Navarro 2013a, 2013b; Bran-Guzmán 2017). 
Tabla 1. Cantidad de asesinatos de personas defensoras de la tierra y el medio ambiente en América Latina para los años 2014, 2015, 2016, 2017 y 2018

\begin{tabular}{ccccccc}
\hline País & \multicolumn{5}{c}{ Año } & \multirow{2}{*}{ Total } \\
\cline { 2 - 6 } & $\mathbf{2 0 1 4}$ & $\mathbf{2 0 1 5}$ & $\mathbf{2 0 1 6}$ & $\mathbf{2 0 1 7}$ & $\mathbf{2 0 1 8}$ & \\
\hline Brasil & 29 & 50 & 49 & 57 & 20 & $\mathbf{2 0 5}$ \\
Colombia & 25 & 26 & 37 & 24 & 24 & $\mathbf{1 3 6}$ \\
Honduras & 12 & 8 & 14 & 5 & 4 & $\mathbf{4 3}$ \\
Perú & 9 & 12 & 2 & 8 & 0 & $\mathbf{3 1}$ \\
Nicaragua & 0 & 12 & 11 & 4 & 0 & $\mathbf{2 7}$ \\
Guatemala & 5 & 10 & 6 & 3 & 16 & $\mathbf{4 0}$ \\
México & 3 & 4 & 3 & 15 & 14 & $\mathbf{3 9}$ \\
Paraguay & 3 & 0 & 0 & 0 & 0 & $\mathbf{3}$ \\
Ecuador & 1 & 0 & 0 & 0 & 0 & $\mathbf{1}$ \\
Costa Rica & 1 & 0 & 0 & 0 & 0 & $\mathbf{1}$ \\
Argentina & 0 & 0 & 0 & 2 & 0 & $\mathbf{2}$ \\
Chile & 0 & 0 & 0 & 0 & 2 & $\mathbf{2}$ \\
República Dominicana & 0 & 0 & 0 & 2 & 0 & $\mathbf{2}$ \\
Venezuela & 0 & 0 & 0 & 1 & 3 & $\mathbf{4}$ \\
\hline Total & $\mathbf{8 8}$ & $\mathbf{1 2 2}$ & $\mathbf{1 2 2}$ & $\mathbf{1 2 1}$ & $\mathbf{8 3}$ & $\mathbf{5 3 6}$ \\
\hline
\end{tabular}

Fuente: Elaboración propia con base en informes de Global Witness 20152016 20172018 y 2019.

En torno a este tipo de conflictualidad, según sostienen diferentes autores, se ha configurado un tipo de antagonismo social en la región (Navarro y Hernández 2010; Bran 2017), como resultado de la rearticulación de un conjunto de actores alrededor de matrices comunitarias en defensa de los territorios y las territorialidades negras, indígenas y campesinas. Frente a ello, el Estado ha contestado mediante diferentes estrategias, una de las cuales ha sido la criminalización de la protesta.

En tal sentido, las mismas organizaciones, los movimientos y las diferentes organismos nacionales e internacionales de derechos humanos, han denunciado el recurso a la criminalización de la protesta y la sistemática violación de los derechos humanos de las comunidades en resistencia contra dicho tipo de (mega)proyectos. Esto ha desembocado en un considerable número de publicaciones alrededor de dicha temática, dándole tanto un tratamiento general como uno específico, basado en estudios de caso, dedicadas a tratar de entender el fenómeno de la criminalización de la protesta y sus manifestaciones características. 
En el caso de Costa Rica, han sido sobre todo las mismas comunidades y los organismos que colaboran con sus procesos, desde donde se ha colocado el tema en discusión. La situación ha redundado en una indefinición conceptual que, según el planteamiento de este trabajo, merece discutirse para tratar de establecer con más precisión de qué se habla cuando se habla de criminalización de la protesta.

De esta manera, el presente trabajo se propone un acercamiento a la temática de la criminalización de la protesta como objeto de estudio de las ciencias sociales, mediante la reconstrucción de un estado de la cuestión. Para tal fin, se enfoca en tres cuestiones consideradas de suma importancia para entender de qué se habla cuando se habla de criminalización de la protesta. En primer lugar, cómo se define esta. En segundo, cuáles son sus manifestaciones más comunes. Y, en tercero, qué función cumple el Estado.

Cabe señalar que, para efectos de este documento, los trabajos consultados no tratan únicamente el caso de la criminalización contra luchas socioterritoriales, sino que se incluyen desde tratamientos generales de la temática hasta consideraciones basadas en casos. Cuando resulte posible, se hará referencia específica a la dinámica de la criminalización en el caso de las luchas socioterritoriales; pero se considera sumamente pertinente contar con un marco más general sobre el tema.

Antes de discutir qué se entiende por criminalización, es necesario hacer un señalamiento respecto de dicho concepto, en relación con la categoría de protesta social. En términos generales, la mayor parte de la literatura que discute el fenómeno coincide en nombrarlo como criminalización de la protesta. En este trabajo, se ha decidido que, para efectos de mantener el diálogo con dicha literatura, se guardara el uso de la misma terminología; no obstante, es importante precisar que, en buena medida, los procesos de criminalización no ocurren durante el ejercicio de la protesta social, entendida esta como acciones no-institucionales mediante las cuales organizaciones, y los movimientos sociales manifiestan su posición contraria en cuanto a un determinado hecho social, económico y político.

La protesta constituye un acto que, generalmente, se inscribe en conflictos sociales multiformes, entre los cuales se desarrollan diferentes formas de acción institucional y no-institucional. Sobre todo en el marco de estos conflictos ocurre la criminalización, por lo que los actos que se les señalan a las personas criminalizadas no siempre se relacionan con su participación en protestas puntuales. Esto no implica que la criminalización de la protesta como tal no ocurra, pues se han documentado diferentes casos de personas procesadas por su participación en acciones de protesta tales como tomas de propiedad o cortes de calle.

\section{La criminalización de la protesta, ¿qué es?}

Tal como ocurre con otros conceptos, el de criminalización de la protesta resulta uno polisémico y que, en muchos casos, se funde (o confunde) con otros como es el caso particular de represión. Esa indefinición conceptual 
produce, en muchos casos, que los estudios sobre el tema terminen calificando como criminalización a un conjunto plural de fenómenos que también podrían encajar en otros conceptos como el mencionado de represión (Favela 2011).

Para efectos de este trabajo, y luego de discutir con otros estudios que han tratado de delimitar el concepto ${ }^{1}$, la criminalización se entenderá fundamentalmente como un proceso consistente en el uso de la represión física y de mecanismos legales y judiciales contra organizaciones y/o movimientos sociales como una forma de control de la protesta social.

El rasgo característico del mencionado proceso sería la llamada judicialización de la protesta, es decir, el uso de la legalidad y la institucionalidad judicial para encausar y procesar a integrantes de organizaciones y movimientos sociales por su participación y acciones en el marco de conflictos y luchas sociales.

No obstante, destacar la dimensión judicial del fenómeno -i.e. la judicialización de la protesta- no supone subordinar la represión, sino resaltar las nuevas funciones que desempeña la institucionalidad judicial en el manejo de la protesta social en la actualidad (Svampa y Pandolfi 2004; Toledo 2007; Artese 2009), en tanto este ha sido el elemento más destacado en la mayoría de la literatura sobre el tema, la cual resalta el uso de los instrumentos legales como un mecanismo de control de la protesta.

Igualmente, pese a que la definición propuesta destaca las dimensiones política y jurídica de la criminalización, un cuerpo de literatura también se ha centrado en estudiar la construcción mediática de la protesta que, en muchos casos, funciona como encuadre para la criminalización. En esta dirección destacan trabajos como los de Artese (2006, 2009), que se enfocan precisamente en el encuadre mediático dado a los cortes de calle (o ruta) registrados en Argentina a finales de la década de 1990, surgidos como un nuevo repertorio de protesta entre las personas desocupadas de distintos pueblos situados en el llamado "interior" argentino. Entre otras cosas, este autor concluye, basado en un trabajo de análisis de notas de prensa escrita, que los medios tendieron a darles una cobertura "condenatoria" a los cortes de ruta y a las personas que participaban en ellos, construyendo un conjunto de representaciones que incluían una identificación de las protestas con la militancia de izquierda, la violencia, el crimen y el delito.

En este proceso, el Estado resulta el actor criminalizador fundamental pues este, mediante su entramado institucional (ejecutivo, legislativo, judicial), genera las condiciones para la criminalización. En ciertos casos, en que el Estado "no se hace presente", otros actores, como el capital privado, ejercen sus funciones, pero comúnmente en articulación con este.

\section{La judicialización de la protesta}

En el estudio de la criminalización de la protesta y, por tanto, en los intentos de definirla conceptualmente, predominan los trabajos que la definen desde
1. Cabe señalar que bastantes estudios consultados discuten conceptualmente el término. Esto representó una dificultad, pues no se contó con un "parámetro" conceptual para dimensionar los fenómenos estudiados en esos trabajos. 
un encuadre legal-constitucional (Gargarella 2008; Bertoni 2010; Zaffaroni 2010). En tal sentido, la mayoría coincide en relacionar la criminalización con el uso del derecho penal contra organizaciones y movimientos sociales. El proceso incluye también la formulación de nueva legislación o la reformulación de la vigente con el fin de crear nuevos tipos penales, que, explícita o implícitamente, encajen conductas típicamente relacionadas con la protesta social.

Es necesario, asimismo, señalar que, en muchos casos, este tipo de medidas riñen con lo establecido en las constituciones políticas y ordenamientos jurídicos nacionales, los cuales protegen, en diferentes grados, el derecho a la protesta mediante la tutela de diferentes libertades. En estos casos, se denotan las tensiones que surgen entre la teoría y la práctica legal-constitucional y el respeto a las libertades constitucionales de las personas, sobre todo cuando estas se manifiestan mediante formas que cuestionan el orden de "lo permitido" (Gargarella 2008; Zaffaroni 2010) y para hacerlo recurren a formatos de acción calificados como contenciosos.

Se trata de definiciones que relacionan la criminalización con la restricción de un conjunto de libertades entre las que se incluyen la de expresión, reunión y tránsito (Gargarella 2008; Bertoni 2010; Zaffaroni 2010). En todos estos casos, como recién se señaló, la reforma legal ha constituido un núcleo central de la criminalización.

Para diferentes autores, se trata de transformar la protesta en un delito sujeto a la jurisdicción judicial y a sus determinantes. Por ejemplo, Toledo (2007) define la criminalización como un "proceso político, mediático y jurídico, que, calificando los actos de protesta como delitos, busca sacar un conflicto social de la arena política para trasladarlo a la arena penal" (p. 262). Palau y Corvalán (2008) y Palau (2009), igualmente destacan la criminalización como un proceso que pretende cambiar la arena de conflicto desde lo político a lo penal. Vázquez coincide con lo anterior, al señalar que la criminalización es un proceso complejo que combina lo político, lo jurídico y lo mediático, con el fin de hacer de la protesta un delito (sin fecha, p. 8). Velazco y Quedena (2015) también entienden la criminalización como un proceso multidimensional.

En la misma línea de lo anterior, se insertan los informes de organismos como la Comisión Interamericana de Derechos Humanos $(\mathrm{CIDH})$, para la cual la criminalización refiere fundamentalmente:

El uso indebido del derecho penal [lo cual] consiste en la manipulación del poder punitivo del Estado por parte de actores estatales y no estatales con el fin de controlar, castigar o limitar el ejercicio del derecho a defender los derechos humanos $(2015,18)$.

En resumen, la criminalización de la protesta se reconoce como un proceso multidimensional, pero en el cual la judicialización se configura como el elemento articulador de una estrategia estatal de control de la protesta social. En términos generales, los conceptos de criminalización y judicialización se tratan como sinónimos, contribuyendo a la confusión conceptual que predomina en los trabajos sobre el tema. 
Precisamente considerando esto, en este trabajo ha preferido distinguir entre represión y judicialización como una forma de precisar dos manifestaciones relacionadas aunque diferentes de lo que se podría considerar como la criminalización de la protesta social. Si bien se pudo sencillamente prescindir de la discusión sobre represión, la cual ha tenido su propia trayectoria teóricoconceptual, lo cierto es que, en muchos casos, la judicialización de la protesta está precedida de un ejercicio de control policial de esta, y en que la represión está presente.

\section{La criminalización de la protesta y sus manifestaciones más comunes}

Los trabajos consultados mencionan un cuantioso número de manifestaciones que se califican indistintamente como criminalización de la protesta. Para este trabajo, y considerando la propuesta conceptual hecha en la sección anterior, se distinguirán dichas manifestaciones según se refieran a la represión o a la judicialización de la protesta.

\section{Las modalidades de la represión}

En el caso de la represión, es típicamente relacionada con la acción de los cuerpos policiales y militares, o bien, de cuerpos parapoliciales y paramilitares, de mucha "tradición" en el ámbito latinoamericano. En este sentido, se podría plantear que la represión consiste, fundamentalmente, en el ejercicio de la violencia, sobre todo física, mediante la acción de cuerpos armados, sean estos legales/formales/regulares o ilegales/informales/irregulares, dirigida contra organizaciones y movimientos sociales que en cierto grado desafían el orden social al recurrir a mecanismos no-institucionales.

En un sentido similar, Earl entiende la represión como la "acción estatal o privada destinada a controlar o restringir la acción no-institucional [i.e. la protesta], incluyendo su inicio" (2011, 263, traducción propia). Su finalidad, continúa la autora, es la de controlar los desafíos no-institucionales contra el poder social, político o cultural.

González (2006), siguiendo a Goldstein, considera la represión como "la acción de gobierno que discrimina brutalmente (sic) a personas o a grupos que se considera que presentan un desafío fundamental a las relaciones de poder..." (Goldstein citado en p. 5). El mismo autor señala tres cuestiones fundamentales de considerar para entender el fenómeno de la represión. En primer lugar, si bien la definición que recupera coloca el énfasis en la acción de los Estados/gobiernos, reconoce que su ejercicio no se limita a estos.

En segundo lugar, sostiene que la represión no debe limitarse a sus manifestaciones de violencia corporal, sino que otras formas de violencia (no-corporal, simbólica), se integran en este fenómeno. Finalmente, e íntimamente relacionado con esto último, González destaca la relación entre este fenómeno y el más general de control social. Según este autor, la represión se inscribe 
2. De acuerdo con González, el uso de la represión tendría tres intenciones: una de disuasión, una de sanción y una de demostración $(2006,8)$.

3. Sobre la relación entre las formas (o repertorios) de protesta y la criminalización, un conjunto de estudios producidos sobre el ciclo de protestas registrado en Argentina entre finales de la década de 1990 y los primeros años de la década de 2000 , muestran el uso particularmente intenso de la criminalización contra los cortes de ruta (o bloqueos) y los actores que los usaban (Svampa y Pandolfi 2004; Artese 2006, 2009). entre los mecanismos de control social y es, en la mayoría de los casos, un recurso de última instancia -i.e. ultima ratio- ${ }^{2}$.

El control policial de las protestas es un tema sistemáticamente trabajado en relación con cómo los cuerpos policiales y, en situaciones excepcionales, los cuerpos militares "contienen" las protestas y cuáles mecanismos usan para ello. Blay (2013) discute este fenómeno para el caso español; señala que el control policial de las protestas tiene un carácter híbrido que combina formas de "gestión negociada", la cual es predominante, con formas de "incapacitación estratégica" (p. 25). Una cuestión fundamental que destaca la autora es que el modelo de control policial de las protestas muestra cambios en relación con los "tiempos, espacios y modos de protesta" (p. 25). Según ella, en el caso de las protestas con un tono marcadamente contencioso (no-institucional), el modelo tiende hacia formas de control más duras, típicas de la incapacitación estratégica.

Lo señalado en Blay (2013) resulta de sumo interés para esta propuesta, pues destaca cómo el control de las protestas, particularmente la represión sobre estas, se distribuye diferencialmente según factores como el tiempo, el espacio y el modo de protestar ${ }^{3}$. No obstante, la autora no menciona que esto también puede cambiar según el actor que protesta.

Cruz (2015), aporta una idea similar en el caso colombiano: detectó que el recurso a la criminalización de la protesta mostraba un comportamiento distinto en el tiempo y el espacio y en función de las características de las protestas y los repertorios en uso. Y, agrega, en Colombia la criminalización se ha ceñido particularmente sobre organizaciones y movimientos de carácter rural, como es el caso de comunidades-territorios negros, indígenas y campesinos.

Según lo identificado en la literatura consultada, muchos de los casos documentados, particularmente para Latinoamérica, se han dirigido contra organizaciones y movimientos socioterritoriales; esto es, actores cuyas luchas y conflictos se concentran en la defensa de sus territorios y bienes comunes. En este sentido, tal como plantea Romo (2008), la criminalización de la protesta tendría un rostro claro que, en su caso, es el de la "clase pobre".

En este trabajo, se sostiene que en América Latina la criminalización de la protesta ha sido particularmente intensa en el caso de las organizaciones y los movimientos socioterritoriales, donde sobre todo participan comunidadesterritorios negros, indígenas y campesinos, quienes mantienen luchas contra proyectos extractivos y neoextractivos que generan desposesión sobre sus territorios y bienes comunes.

En esta misma dirección, el trabajo de Poulos y Haddad (2016) demuestra que la represión de este tipo de movimientos es un fenómeno identificable en diferentes Estados/gobiernos, indiferentemente de sus sistemas políticos -i.e. democráticos, semidemocráticos, no democráticos- y que esta es más frecuente en conflictos que incluyen lo que ellas denominan como grupos marginados (la clase pobre en Romo) que luchan contra proyectos extractivos (y neoextractivos, agregaría) en sus comunidades (territorios). 


\section{Las modalidades de la judicialización}

Tal como se señaló en la definición de criminalización propuesta en este trabajo, la dimensión judicial -o judicialización- de dicho fenómeno, es seguramente su rasgo característico y lo que la diferencia de la "mera" represión. La judicialización de la protesta consiste, principalmente, en el uso de los instrumentos legales y de las instituciones judiciales como un mecanismo de control sobre las organizaciones y los movimientos sociales. Se trata, como señalan diferentes autores en sus definiciones, de judicializar lo político o de politizar lo judicial, mediante el traslado de los conflictos sociales de la arena política a la arena judicial (Toledo 2007; Palau y Corvalán 2008; Palau 2009).

Para cumplir ese propósito, los Estados/gobiernos recurren a los instrumentos legales como un mecanismo de control sobre los movimientos. En ciertos casos, cuando no cuentan con los instrumentos, crean nueva legislación, tanto ejecutiva -i.e. decretos y similares- como legislativa, con el fin de introducir lo necesario para posibilitar la judicialización de ciertos tipos de conductas típicamente relacionadas con la acción de los movimientos sociales.

De manera que el Estado crea un marco legal-judicial que posibilita el procesamiento y encausamiento legal de militantes de organizaciones y movimientos sociales por precisamente participar en estos. Reformas constitucionales y, sobre todo, de instrumentos específicos como los códigos penales, constituyen una práctica común en el marco de las políticas de criminalización en América Latina.

Una modalidad destacada en diferentes trabajos ha consistido en crear marcos políticos y legales para etiquetar conductas que, en principio, no se relacionarían con la participación en protestas sociales, pero que, en la práctica, terminan siendo recurridos para este fin, como han sido las legislaciones "anti-terroristas" en América Latina, encuadradas en las políticas de seguridad nacional de los Estados. Un ejemplo es el Estado chileno que recurrentemente ha usado dicha legislación anti-terrorista para procesar a militantes mapuche (Toledo 2007; Fernández y Ojeda 2015). Similar ha ocurrido en Ecuador (Pérez y Solís 2014), donde una importante cantidad de militantes de organizaciones y movimientos sociales han sido procesados bajo el parámetro de dicha legislación. Lo mismo ha sido señalado para los casos salvadoreño (Hernández 2009) y colombiano (Uprimny y Sánchez 2010; Cruz 2015), este último en el marco de un conflicto armado que ha creado condiciones particulares para la criminalización de la protesta.

En países como Bolivia (Rodríguez y Rojas 2010) y México (Romo 2008), el narcotráfico ha sido también recurrido como un marco justificador para políticas que han terminado siendo usadas para criminalizar la protesta social (Rábago 2010).

Asimismo, los estudios mencionan la relación entre el incremento de la criminalización de la protesta y la proliferación de cierto tipo de repertorios de carácter no-institucional como los bloqueos (o cortes de ruta) (Svampa y Pandolfi 2004; Artese 2006, 2009) o los diferentes actos contra la propiedad -pública o privada- (Pérez y Solís, 2014) como la población mapuche (Toledo 
2007; Fernández y Ojeda 2015). Mediante la contraposición de derechos, el Estado y la institucionalidad han construido el bloqueo como un delito que en muchos casos es castigado penalmente, incrementando el costo potencial de participar en bloqueos.

Pero, sin duda, la modalidad más común de judicialización de la protesta es el procesamiento y encausamiento legal de militantes, lo cual está íntimamente relacionado con lo recién mencionado. En prácticamente toda la región latinoamericana, se han creado y modificado tipos penales que, de manera directa o indirecta, buscada o no, han terminado calificando conductas comunes de las organizaciones y los movimientos sociales, en delitos.

Tabla 2. La participación de las esferas ejecutiva y judicial en la criminalización de la protesta

\begin{tabular}{|c|c|c|c|}
\hline Esfera & Actores & Función & Acciones \\
\hline Ejecutiva & $\begin{array}{l}\text {-Cuerpos } \\
\text { policiales. } \\
\text {-Cuerpos } \\
\text { militares. } \\
\text {-Organismos } \\
\text { de } \\
\text { inteligencia. } \\
\text {-Otras } \\
\text { instituciones } \\
\text { específicas. }\end{array}$ & $\begin{array}{l}\text { Represión } \\
\text { Ejercicio de la } \\
\text { violencia física. } \\
\text { Criminalización } \\
\text { de hecho. }\end{array}$ & $\begin{array}{l}\text {-Negación y deslegitimación de los actores } \\
\text { sociales y de su condición de interlocutores } \\
\text { legítimos. }\end{array}$ \\
\hline Judicial & $\begin{array}{l}\text {-Poder } \\
\text { Judicial } \\
\text {-Jueces } \\
\text {-Fiscales }\end{array}$ & $\begin{array}{l}\text { Judicialización } \\
\text { Criminalización } \\
\text { de derecho. }\end{array}$ & $\begin{array}{l}\text {-Fortalecimiento y redefinición de las funciones } \\
\text { de los cuerpos policiales. } \\
\text {-Uso de los cuerpos policiales, militares o } \\
\text { paramilitares para reprimir. } \\
\text {-Creación de nueva o modificación de } \\
\text { legislación penal (creación o modificación de } \\
\text { nuevos tipos penales, incrementos de penas). } \\
\text {-Limitación de derechos a la libertad de } \\
\text { expresión, reunión, asociación, petición, etc., } \\
\text { como forma de limitar el derecho a la protesta. } \\
\text {-Contraposición de derechos (libertad de } \\
\text { tránsito vs libertad de expresión/derecho a la } \\
\text { protesta). } \\
\text {-Creación de marcos justificadores (seguridad } \\
\text { nacional, guerra contra el terrorismo, } \\
\text { narcotráfico). } \\
\text {-Construcción de los actores como criminales } \\
\text { (mediante medios de comunicación). } \\
\text {-Denuncias y juicios penales. }\end{array}$ \\
\hline
\end{tabular}

Fuente: Elaboración propia con base en Della Porta (1999), Svampa y Pandolfi (2004), Toledo (2007), Gargarella (2008), Romo (2008), Palau y Corvalán (2008), Artese (2006, 2009), Hernández (2009), Palau (2009), Bertoni (2010), Rábago (2010), Uprimny y Sánchez (2010), Valle (2010), Zaffaroni (2010), Earl (2011), Pérez y Solís (2014), Cruz (2015), Fernández y Ojeda (2015), Velazco y Quedena (2015), Poulos y Haddad (2016). 
Esto ha permitido que tanto el Estado como otros actores interesados en contrarrestar el ejercicio de la protesta social, como las empresas nacionales y transnacionales, recurran a dicha legislación para demandar a militantes bajo diferentes figuras jurídicas. Tal como diferentes estudios han señalado, en muchos casos, el fin último de esta acción no es conseguir una condena, sino desarticular la protesta social.

Finalmente, la tabla 2 muestra de manera sintética la participación de las esferas ejecutiva y judicial y sus acciones en los procesos de criminalización.

\section{El Estado como actor criminalizador}

Una mayoría de los estudios consultados coincide en identificar el Estado como el actor determinante en la producción de las políticas de criminalización de la protesta social (Svampa y Pandolfi 2004; Toledo 2007). Para ello, el Estado y su complejo entramado político-institucional aplican diferentes mecanismos orientados hacia la creación de las condiciones políticas y judiciales necesarias para la criminalización de militantes de organizaciones y movimientos sociales.

En ese sentido, un primer elemento para destacar es la participación de actores institucionales de las tres esferas de poder estatal: el ejecutivo, el legislativo y el judicial. Consistente con lo señalado en la sección anterior, la multiformidad de las políticas de criminalización requiere la participación de diversos actores institucionales en su diseño y ejecución. Cada uno de ellos desempeña una función específica, pero generalmente articulada a una estrategia política con un fin común.

No obstante, diferentes estudios coinciden en que los Estados no mantienen una sola política de criminalización y que esta cambia en función de un espacio-tiempo determinado y de las acciones, los actores y las demandas que los desafían. El trabajo de Della Porta (1999), si bien está centrado en la represión policial contra las protestas sociales en Italia y Alemania durante el período 1950-1990, introduce importantes consideraciones sobre las diferentes formas e intensidades que este fenómeno cobra. Para la autora, la represión es un barómetro de las condiciones que enfrentan las organizaciones y los movimientos sociales en los entornos políticos donde se desarrollan, particularmente sobre cómo reacciona el Estado y qué está dispuesto a hacer y qué no para controlar la protesta social en un momento determinado. A tal finalidad, la autora distingue entre 1) un control represivo y uno norepresivo/tolerante; 2) una represión selectiva y una difusiva; 3) un control preventivo y uno reactivo; 4) un control duro y uno blando; y, 5) tácticas legales e ilegales (sucias, las llama Della Porta).

De esta manera, sostiene, los Estados y, particularmente, los cuerpos policiales recurren a diferentes combinaciones de estos factores para controlar la protesta social. Asimismo, Della Porta propone un modelo de tres niveles para estudiar la represión, el cual permite considerar la complejidad de factores que participan en la determinación de las formas de control policial de las protestas. En primer lugar, introduce un nivel general de análisis centrado en 
4. En oposición a una política difusiva de criminalización de la protesta social, en los términos que lo han planteado autoras como Della Porta (citada en González 2006). las características institucionales y culturales que puede incidir en la represión. El segundo es un nivel intermedio que refiere a lo que la autora denomina como la configuración de poder, la cual contempla factores de orden coyuntural como los reordenamientos político-partidarios y de gobierno. Finalmente, un tercer nivel de orden específico se halla relacionado con la cierta autonomía que manejan las mismas burocracias policiales para definir y, principalmente, ejecutar la represión policial.

Por su parte, otros trabajos se han centrado en el par represión selectiva o difusiva y sostienen que la criminalización es, fundamentalmente, selectiva ${ }^{4}$ (Romo 2008; Rodríguez y Rojas 2010; Blay 2013; Vázquez sin fecha); es decir, no se ciñe de la misma manera ni con la misma intensidad contra todos los actores sociales ni en todos los momentos de la protesta. Por el contrario, como primer elemento, la criminalización incide particularmente entre aquellos actores que cuentan con menores recursos -i.e. económicos, políticos, legales, etc.- (Romo 2008; Poulos y Haddad 2016). Asimismo, autoras como Poulos y Haddad (2016) han dicho que la criminalización en sus diferentes manifestaciones es más común en el caso de movimientos de tipo socioterritorial, cuyas luchas se concentran en la defensa de sus territorios y bienes comunes frente a proyectos de tipo extractivo y neoextractivo.

Un segundo elemento se relaciona con la participación estatal que pasa precisamente por la inacción frente a conflictos entre actores. En ciertos casos, el Estado no acciona directamente en un conflicto ni en su criminalización, pero permite que otros actores, generalmente los capitales privados ejerzan diferentes formas de violencia física y no-física contra comunidades organizadas para combatir proyectos de distinto tipo. En este caso, también es posible pensar en una acción estatal precedente que es la de crear las condiciones político-institucionales y legales para la criminalización, lo cual se relaciona directamente con lo indicado en el primer punto o en el incumplimiento de sus obligaciones como Estado para proteger los derechos humanos y la seguridad de las organizaciones y los movimientos sociales.

Un tercer elemento refiere a la ubicuidad de las políticas de criminalización "indiferentemente" de los tipos de sistema político. Se trate de sistemas democráticos, semidemocráticos o no-democráticos/autoritarios, la criminalización forma parte de las estrategias estatales para el control de las protestas y de los conflictos sociales.

Ciertamente, los diferentes estudios ofrecen información contrastante en términos de la incidencia e intensidad de la criminalización según el tipo de sistema. Por ejemplo, Poulos y Haddad (2016) demostraron en su estudio que el uso de la represión violenta contra los movimientos sociales no es un fenómeno único de los Estados no-democráticos. De acuerdo con las autoras, los resultados de su trabajo muestran que la represión violenta contra grupos sociales marginados enfrascados en protestas socioambientales es un serio problema para todos los países, indiferentemente de su tipo de sistema político. 
González (2006) expresa que el uso de la represión puede resultar particularmente común en el caso de sistemas semidemocráticos, pues en estos se presentan condiciones suficientes para no desestimular la protesta, aunque también la posibilidad de que esta se conteste con violencia. A criterio del autor, en sistemas no-democráticos, la violencia es tanta que tiende a la no protesta. Mientras en el caso de los democráticos "no se produce mucho conflicto violento, porque la mayoría de los grupos pueden conseguir sus intereses mediante canales más pacíficos y menos costosos en términos de participación política" (p. 16).

Una cuestión similar se podría señalar en términos de la orientación políticoideológica de los gobiernos. En el caso latinoamericano, algunos trabajos han indicado la continuidad de las políticas de criminalización, incluso durante los llamados gobiernos progresistas (o neodesarrollistas) en Suramérica (ver por ejemplo Svampa y Pandolfi 2004, para el caso argentino; y Pérez y Solís 2014, para el caso ecuatoriano).

Precisamente, Pérez y Solís hablando sobre el caso ecuatoriano mencionan que:

Si bien la criminalización de la protesta ha sido histórica en todos los gobiernos, la revolución ciudadana [de Correa] ha intensificado la represión. La condena a los Diez de Luluncoto tiene por ello implicaciones mayores. Con este caso se institucionaliza la judicialización de la organización social $(2014,165)$.

Pero, en consonancia con lo indicado en las secciones anteriores, sin duda el elemento característico de la participación estatal en las políticas de criminalización actuales es el redimensionamiento de las funciones judiciales o la judicialización de la política, lo cual consiste, como se ha señalado, en el traslado de los conflictos sociales desde esa arena a la judicial, con el fin transformar el problema en uno de índole penal (Svampa y Pandolfi 2004; Toledo 2007; Artese, 2009).

Tal como sostienen diferentes autores, bajo el dominio de gobiernos autoritarios y dictatoriales el recurso a lo legal resulta innecesario pues la legitimidad emana de la autoridad de un líder, el cual fija los parámetros de lo punible y lo no punible. Por el contrario, en gobiernos democráticos o que se presentan como tales, resulta necesario construir las fuentes de legitimidad, las cuales incluyen la legitimidad para castigar. Dichas fuentes son buscadas en el marco legal como una ruta para dotar de legitimidad a las acciones estatales, en este caso las orientadas hacia el control de la protesta social (Favela 2011).

En dicho proceso, el Estado hace uso de diferentes estrategias para construir la legitimidad, entre las que se incluyen la producción de los instrumentos legales y marcos institucionales necesarios (Svampa y Pandolfi 2004; Toledo 
2007; Favela 2011) y también la construcción mediática de las organizaciones y los movimientos sociales como criminales (Artese 2009).

De la misma manera, se fabrican discursos orientados a contraponer derechos, como, por ejemplo, las "polémicas" entre el derecho a circular y el derecho a protestar, en el caso particular de los bloqueos (o cortes de ruta), como una forma de obtener legitimidad mediante el apoyo de la sociedad y de grupos de interés a las medidas de criminalización (Svampa y Pandolfi 2004; Gargarella 2008; Artese 2009).

También se denota una tensión entre la "teoría" y la "práctica" estatal en torno a la tutela y protección de los derechos relacionados con el ejercicio de la protesta social (Gargarella 2008; Rábago 2010; Zaffaroni 2010).

Ahora bien, un elemento menos destacado en la literatura consultada refiere a las acciones estatales: a pesar de que no están directamente orientadas a la criminalización, terminan desembocando en conflictos que generan ese tipo de situaciones. Más concretamente, es primordial considerar que, en el caso particular de América Latina, los Estados en conjunto con actores noestatales, particularmente empresas extractivas nacionales y extranjeras, han impulsado modelos de acumulación basados en la desposesión de los territorios y los bienes comunes de muchas comunidades urbanas y, sobre todo, rurales, generado que estas mismas comunidades desarrollen procesos de resistencia en el marco de los cuales resultan criminalizadas de diferentes formas.

Tales situaciones tienden a incrementarse en aquellos casos que ocurren en los "márgenes de los Estados", donde el control político y territorial recae más en los actores no-estatales (empresas, cárteles, etc.) y menos en los estatales. En situaciones así, la "falta de Estado" se configura ella misma como un factor que propicia la criminalización de la protesta, en tanto se constituyen como territorios donde la ley es determinada por actores extraestatales.

Entonces, es necesario profundizar en el tipo de relaciones que se configuran entre los actores estatales y no-estatales en el marco de los procesos de criminalización, pues, en general, la mayor parte de la literatura se limita a enunciarlas, dando como supuesto que, en todos los casos, las relaciones son simios lares. Pese a esto, un ejercicio de este tipo trasciende las posibilidades de este trabajo.

Igualmente indispensable es profundizar son las relaciones entre el Estado y los actores criminalizados; es decir, las organizaciones y los movimientos sociales. Aunque la literatura sobre el tema se concentra en destacar la participación directa e indirecta de los Estados en la criminalización, lo cierto es que el Estado se configura como un terreno de conflicto entre diferentes actores. En ese sentido, los actores criminalizados también interpelan a los actores estatales para que actúen en el sentido de proteger la labor de defensa de derechos humanos y a las personas que participan en organizaciones y movimientos sociales. Este hecho constata el carácter complejo y contradictorio que encarna el entramado político-institucional, también sujeto a las di- 
námicas políticas coyunturales que pueden cambiar las condiciones en las cuales un determinado Estado puede controlar el conflicto social.

\section{Cierre}

Luego de lo discutido en este trabajo, es posible señalar una serie de elementos en cuanto a lo que se entiende como criminalización de la protesta y algunas de sus características más destacadas, con el fin de contribuir a delimitar conceptualmente la criminalización y a un tratamiento más preciso de lo que se pueda considerar/calificar como tal.

En primer lugar, la criminalización es un fenómeno multidimensional, que articula una dimensión política -i.e. represión-, una jurídica y una mediática. Lo particularmente característico de lo que comúnmente se denomina como criminalización es lo que la literatura consultada denomina como judicialización.

Segundo, la judicialización refiere fundamentalmente a un proceso mediante el cual se intenta trasladar un conflicto de la arena política a la judicial, para lo cual se recurre a los instrumentos legales y a las instituciones judiciales.

Tercero, la criminalización es un fenómeno diferencialmente experimentado según tiempos, espacios, formas y actores de la protesta. En tal sentido, la criminalización se inscribe en una estrategia más general de control de las protestas sociales, y en que los Estados recurren a diferentes mecanismos para dicho fin. La criminalización no es necesariamente el primero ni el único mecanismo estatal de control de la protesta.

Cuarto, en el caso latinoamericano, los llamados movimientos socioterritoriales son los actores más criminalizados. En relación con el punto anterior, la literatura consultada sugiere que cierto tipo de organizaciones y movimientos sociales experimentan con más intensidad y frecuencia la criminalización de sus luchas. Esa situación podría relacionarse tanto con el tipo de conflicto y lo que está en juego (es decir, con cómo el conflicto que se plantea cuestiona o no las estructuras económicas y políticas de una sociedad en un momento determinado) como con los recursos con que las organizaciones y los movimientos disponen para protestar y, en última instancia, enfrentar la criminalización de la protesta.

Quinto, el Estado es un actor central en los procesos de criminalización, sea mediante el ejercicio de la violencia física/corporal, pero también mediante el de otras formas de violencia no-corporal o la producción de legislación penal que criminalice a organizaciones y/o movimientos sociales. Asimismo, en ciertos casos el Estado no es el actor criminalizador directo, pero sí participa indirectamente al permitirles el ejercicio de la violencia a otros actores como los capitales privados -nacionales y transnacionales-. 


\section{Bibliografía}

Artese, Matías. 2006. El discurso que criminaliza. Un estudio sobre la construcción social de la deslegitimación de la protesta (1996-1999). Documento de Jóvenes Investigadores, 13.

Artese, Matías. 2009. "Criminalización de la protesta en Argentina. Una construcción de lo delictivo más allá de la esfera jurídica». América Latina Hoy, 52, 149-169.

Bertoni, Eduardo. 2010. ¿Es legítima la criminalización de la protesta social? Derecho penal y libertad de expresión en América Latina. Buenos Aires: Universidad de Palermo.

Blay, Ester. 2013. «El control policial de las protestas en España». InDret Revista para el Análisis de Derecho.

Bran-Guzmán, Emanuel. 2017. «Conflictividad socioambiental en Centroamérica. Una década de rearticulación y movilización social y política». Argumentos, 30 (83), 43-68.

Comisión Interamericana de Derechos Humanos. 2015. Criminalización de la labor de las defensoras y defensores de derechos humanos. Washington: OEA.

Cruz, Edwin. 2015. «El derecho a la protesta social en Colombia». Pensamiento Jurídico, 42, 47-69.

Della Porta, Donatella. 1999. «Movimientos sociales y Estado: algunas ideas en torno a la represión policial de la protesta». En Movimientos sociales: perspectivas comparadas, editado por Doug McAdam, John McCarthy y Mayer Zald Madrid: Istmo.

Earl, Jennifer. 2011. «Political Repression: Iron Fists, Velvet Gloves, and Diffuse Control». Annual Review of Sociology, 37, 261-284.

Favela, Margarita. 2011. Ampliación de la ciudadanía y criminalización de la protesta: negación de derechos políticos y reducción de Estado de Derecho. Ponencia presentada en las Jornadas Anuales de Investigación, CEIICH-UNAM. 
Fernández, Francisca y Ojeda, Doris. 2015. «Criminalización de la resistencia mapuche como política del miedo». Athenea Digital, 15 (4), 267-277. Recuperado de http://atheneadigital.net/article/view/v15-n4-fernandezojeda/1595-pdf-es

Gargarella, Roberto. 2008. «El derecho frente a la protesta social». En Revista de la Facultad de Derecho de México, 58 (250), 183-199.

González, Eduardo. 2006. «Sobre el concepto de represión». Hispania Nova. Revista de Historia Contemporánea, 6.

Global Witness. 2015. ¿Cuántos más? El medio ambiente mortal de 2014.

Global Witness. 2016. En terreno peligroso. El medio ambiente mortal de 2015.

Global Witness. 2017. Defender la tierra. El medio ambiente mortal de 2016.

Global Witness. 2018. ¿A qué precio? El medio ambiente mortal en 2017.

Global Witness. 2019. Enemies of the State?

Harvey, David. 2004. El nuevo imperialismo: acumulación por desposesión. Buenos Aires: CLACSO.

Hernández, Josué. 2009. La criminalización de la protesta pública en El Salvador, una forma de violación a derechos fundamentales. El Salvador: Instituto Americano de Educación Superior.

Modonesi, Massimo. 2010. Subalternidad, antagonismo, autonomía: marxismos y subjetivación. Argentina: CLACSO, Prometeo Libros.

Modonesi, Massimo. 2016. El principio antagonista. México: Editorial Ítaca.

Navarro, Mina y Pineda, César. 2009. «Luchas socioambientales en América Latina y México. Nuevas subjetividades y radicalidades en movimiento». Revista Bajo El Volcán, 8 (14), 81-104.

Navarro, Mina y Hernández, Oliver. 2010. «Antagonismo social en las luchas socioambientales en México: cuerpo, emociones y subjetividad como terreno de lucha contra la afectación». Revista Latinoamericana de Estudios sobre Cuerpos, Emociones y Sociedad, 2 (4), 77-92.

Navarro, Mina. 2013a. «Las luchas indígenas y campesinas contra el despojo capitalista en México: subjetividades políticas en la defensa y gestión de los bienes comunes naturales». Boletín Onteaiken, 15, 71-84. 
Navarro, Mina. 2013b. «Subjetividades políticas contra el despojo capitalista de bienes naturales en México». Acta Sociológica, 62, 135-153.

Palau, Marielle y Corvalán, Ramón. 2008. «Criminalización de movimientos sociales en Paraguay: algunos elementos para comprender su magnitud». En Criminalización de la protesta y de los movimientos sociales, organizado por Kathrin Buhl y Claudia Korol. Sao Paulo: Fundación Rosa Luxemburgo.

Palau, Marielle. 2009. «Represiones, atropellos y persecuciones a comunidades y organizaciones campesinas». En Criminalización a la lucha campesina, coordinado por Marielle Palau, Juan Martens y José Sánchez. Paraguay: BASE IS.

Pérez, Carlos y Solís, Fernanda. 2014. «Territorio, resistencia y criminalización de la protesta». En Varios Autores. La restauración conservadora del correísmo. Ecuador.

Poulos, Helen and Haddad, Mary Alice. 2016. «Violent repression of environmental protests». Springer Open Journal.

Rábago, Miguel. 2010. «La criminalización de la protesta social como forma de restricción de la libertad de expresión en México: movimientos sociales en el centro de la llamada "lucha contra la delincuencia organizada"». En ¿Es legítima la criminalización de la protesta social? Derecho penal y libertad de expresión en América Latina, compilado por Eduardo Bertoni. Buenos Aires: Universidad de Palermo.

Rodríguez, Eduardo y Rojas, Farit. 2010. «Criminalización y derecho a la protesta». En ¿Es legítima la criminalización de la protesta social? Derecho penal y libertad de expresión en América Latina, compilado por Eduardo Bertoni. Buenos Aires: Universidad de Palermo.

Romo, Pablo. 2008. «La criminalización de la protesta social en México». En Criminalización de la protesta y de los movimientos sociales, organizado por Kathrin Buhl y Claudia Korol. Sao Paulo: Fundación Rosa Luxemburgo.

Svampa, Maristella y Pandolfi, Claudio. 2004. «Las vías de la criminalización de la protesta en Argentina». En OSAL, 5 (14), 285-296. 
Toledo, Víctor. 2007. «Prima ratio. Movilización mapuche y política penal. Los marcos de la política indígena en Chile 1990-2007». OSAL, 22, 253275.

Uprimny, Rodrigo y Sánchez, Luz. 2010. «Derecho penal y protesta social». En ¿Es legítima la criminalización de la protesta social? Derecho penal y libertad de expresión en América Latina, compilado por Eduardo Bertoni. Buenos Aires: Universidad de Palermo.

Valle, Dayra. 2010. «Criminalización de la protesta social en Nicaragua como forma de restricción a la libertad de expresión». En ¿Es legítima la criminalización de la protesta social? Derecho penal y libertad de expresión en América Latina, compilado por Eduardo Bertoni. Buenos Aires: Universidad de Palermo.

Vázquez, Mirtha. sin fecha. La criminalización de la protesta social como estrategia de desarticulación de movimiento social en Perú. Documento de Trabajo.

Velazco, David y Quedena, Rosa. 2015. La criminalización de la protesta y el caso Majaz. Perú: OXFAM.

Zaffaroni, Raúl. 2010. «Derecho penal y protesta social». En ¿Es legítima la criminalización de la protesta social? Derecho penal y libertad de expresión en América Latina, compilado por Eduardo Bertoni. Buenos Aires: Universidad de Palermo. 